\title{
Solid-Phase Silicon Homoepitaxy via Shear-Induced Amorphization and Recrystallization
}

\author{
Thomas Reichenbach $\odot,{ }^{1,2}$ Gianpietro Moras $\odot,{ }^{1,{ }^{*}}$ Lars Pastewka $\odot,{ }^{1,3,4,5}$ and Michael Moseler $\oplus^{1,2,4,5}$ \\ ${ }^{1}$ Fraunhofer IWM, MicroTribology Center $\mu$ TC, Wöhlerstraße 11, 79108 Freiburg, Germany \\ ${ }^{2}$ Institute of Physics, University of Freiburg, Hermann-Herder-Straße 3, 79104 Freiburg, Germany \\ ${ }^{3}$ Department of Microsystems Engineering, University of Freiburg, Georges-Köhler-Allee 103, 79110 Freiburg, Germany \\ ${ }^{4}$ Freiburg Materials Research Center, University of Freiburg, Stefan-Meier-Straße 21, 79104 Freiburg, Germany \\ ${ }^{5}$ Cluster of Excellence livMatS, Freiburg Center for Interactive Materials and Bioinspired Technologies, \\ University of Freiburg, Georges-Köhler-Allee 105, 79110 Freiburg, Germany
}

(Received 9 March 2021; accepted 16 July 2021; published 16 September 2021)

\begin{abstract}
We study mechanically induced phase transitions at tribological interfaces between silicon crystals using reactive molecular dynamics. The simulations reveal that the interplay between shear-driven amorphization and recrystallization results in an amorphous shear interface with constant thickness. Different shear elastic responses of the two anisotropic crystals can lead to the migration of the amorphous interface normal to the sliding plane, causing the crystal with lowest elastic energy density to grow at the expense of the other one. This triboepitaxial growth can be achieved by crystal misorientation or exploiting elastic finite-size effects, enabling the direct deposition of homoepitaxial silicon nanofilms by a crystalline tip rubbing against a substrate.
\end{abstract}

DOI: 10.1103/PhysRevLett.127.126101

Silicon crystals have intriguing, size-dependent, anisotropic mechanical properties with diverse brittle and ductile deformation mechanisms [1-5] that are responsible for the failure of micro- and nanodevices but can also be harnessed to improve their manufacturing and performance. For instance, understanding and controlling brittle fracture propagation at the atomic scale is crucial to produce silicon surfaces with controlled roughness [6] or atomic smoothness [7], while exploiting the transition to a dislocationmediated ductile behavior at the nanoscale can help improve the mechanical reliability and strain engineering of silicon devices [8]. An alternative way to accommodate plastic deformation in crystalline silicon is the formation of amorphous shear bands $[4,9,10]$. So far, shear-induced amorphization has mainly been studied for its role in wear processes [11,12]. Interestingly, however, our recent molecular dynamics (MD) simulations [10] reveal that the plastic shear deformation that drives the crystallineto-amorphous transition at the boundaries of a shear band simultaneously enables a competing amorphous-to-crystalline transition that could potentially be exploited for homoepitaxial crystal growth [13,14].

Simulations [10] show that a nanoscale amorphous silicon $(a-\mathrm{Si})$ layer forms at the sliding interface between two diamond-cubic silicon crystals. Here, amorphization is

Published by the American Physical Society under the terms of the Creative Commons Attribution 4.0 International license. Further distribution of this work must maintain attribution to the author(s) and the published article's title, journal citation, and DOI. a mechanically driven, nonequilibrium process that enables the localization of the shear deformation within an $a-\mathrm{Si}$ sliding region with comparatively low shear strength $[10,11]$. Simultaneously, plastic shear deformation provides the atomic mobility necessary to overcome the energy barriers for the thermodynamically driven recrystallization of metastable $a-\mathrm{Si}$, which would be otherwise kinetically hindered at temperatures well below the melting point [10]. The competition between amorphization and recrystallization at the amorphous-crystal interfaces delimiting the $a$-Si region results in a constant thickness of the $a$-Si layer, whose position changes stochastically with no unidirectional drift if the two crystals have identical size and orientation. In this Letter, we use MD simulations to gain further insights into the competition between shear-induced amorphization and recrystallization and to explore conditions that render the movement of the amorphous layer unidirectional, thus leading to the growth of one crystal at the expense of the other one.

We find that this tribologically induced homoepitaxial process is possible if the two sliding crystals have different crystallographic orientations, and we name it "triboepitaxy," a combination of nanotribology and solid-phase epitaxy [15]. We show that, for a variety of surface orientations, the growth direction is determined by the elastic energy per atom $E_{\mathrm{el}}$ : the crystal with lowest $E_{\mathrm{el}}$ grows. Hence, triboepitaxial growth can be controlled by exploiting elastic anisotropy, i.e., by tuning the relative orientation of the two crystals with respect to the sliding direction. Finally, we propose a model experiment that could serve as a validation of the triboepitaxy concept and 

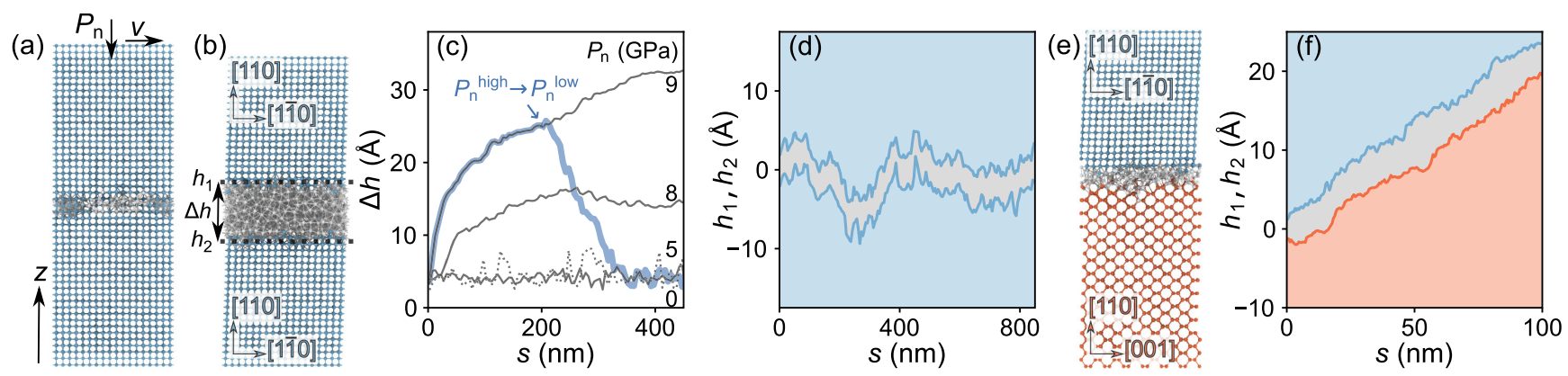

FIG. 1. Two diamond-cubic Si(110) crystals in relative motion. (a) Interface under normal load $P_{n}$ before sliding along the [1 $\left.1 \overline{1} 0\right]$ direction with $v=10 \mathrm{~ms}^{-1}$. (b) After shearing at $P_{n}=8 \mathrm{GPa}$ for a sliding distance $s=300 \mathrm{~nm}$. Blue and gray spheres represent Si atoms in the crystalline and amorphous regions, respectively. (c) $a$-Si thickness $\Delta h\left(s, P_{n}\right)$ as a function of $s$ for different $P_{n}$ (dotted line, $P_{n}=0$ ). The blue line shows $\Delta h(s)$ when the initial $P_{n}^{\text {high }}=9 \mathrm{GPa}$ is reduced to $P_{n}^{\text {low }}=5 \mathrm{GPa}$ at $s=200 \mathrm{~nm}$. (d) Vertical positions $h_{1}(s)$ and $h_{2}(s)$ of the two amorphous-crystal interfaces for $P_{n}=5 \mathrm{GPa}$. Amorphous and crystalline regions are gray and blue, respectively. (e) Shearing of differently oriented crystals: The upper crystal slides along the [110] and the lower crystal (in red) along the [001] direction. (f) $h_{1}(s)$ and $h_{2}(s)$ for the system in (e) with $P_{n}=0$.

stimulate research on the viability of novel mechanical scanning-probe nanolithography techniques $[16,17]$ for the deposition of crystalline silicon nanostructures. This approach would require neither surface nanopatterning to precisely control the location, shape, and size of the grown crystalline structures $[13,14]$ nor elevated temperatures [15] or other chemical elements to catalyze crystallization [13]. In such an experimental setup, triboepitaxial growth could also be controlled by exploiting elastic finite-size effects, e.g., by tuning shape and size of the writing tip.

We perform classical reactive MD simulations using the screened version [18] of the empirical bond-order potential by Kumagai et al. [19] to study the evolution of a shearinduced $a$-Si phase between two diamond-cubic silicon crystals [10,11]. In the Supplemental Material [20], we show that the Stillinger-Weber potential [29] and a machine-learning-based Gaussian approximation potential [30,31] yield comparable results. Figure 1(a) displays the interface between two silicon (110) surfaces before sliding along the $[1 \overline{1} 0]$ direction [periodic boundary conditions are applied in the sliding plane; analogous simulations for (001) surfaces are described in the Supplemental Material [20] ]. After imposing an external normal pressure $P_{n}$ and thermalizing the system to $T=300 \mathrm{~K}$ by means of specially tailored barostat and thermostat [11], a top layer of atoms is rigidly moved at a constant velocity $v=$ $10 \mathrm{~ms}^{-1}$ along the sliding direction, while a bottom layer is kept fixed (see Supplemental Material [20] for computational details).

Upon sliding, an $a$-Si layer forms [snapshot in Fig. 1(b)] at the interface between the two crystals. It entirely accommodates the plastic shear deformation of the system [10], and its thickness $\Delta h\left(s, P_{n}\right)$ increases with sliding distance $s$ and with applied normal pressure $P_{n}$ [Fig. 1(c) for $P_{n}=0-9 \mathrm{GPa}$; see Supplemental Material [20] for details of $\Delta h$ computation]. As previously reported [10], the crystalline-to-amorphous transition is mechanically induced, local temperatures remain below $400 \mathrm{~K}$, and a melting transition can be excluded. Interestingly, as the sliding distance increases, $\Delta h\left(s, P_{n}\right)$ saturates at a constant value $\Delta h_{\mathrm{eq}}\left(P_{n}\right)$. Since mechanical amorphization alone would lead to a strict monotonic increase of $\Delta h[10,11]$, saturation indicates the presence of a competing process, namely, shear-induced recrystallization [10]. Indeed, the vertical positions $\left(h_{1}, h_{2}\right)$ of the lower and upper amorphous-crystal interfaces fluctuate with constant $h_{2}-h_{1}=$ $\Delta h$ and no clear drift [Fig. 1(d)].

Further evidence of the recrystallization process is provided by the blue line in Fig. 1(c). Here, we perform a sliding simulation at high pressure $\left(P_{n}^{\text {high }}=9 \mathrm{GPa}\right)$ and suddenly decrease the normal pressure to $P_{n}^{\text {low }}=5 \mathrm{GPa}$ at a sliding distance $s_{P_{n}^{\text {high }} \rightarrow P_{n}^{\text {low }}}=200 \mathrm{~nm}$. This causes a rapid decrease of the $a$-Si thickness from $\Delta h\left(s_{P_{n}^{\text {high }} \rightarrow P_{n}^{\text {low }}}, P_{n}^{\text {high }}\right)=$ $2.5 \mathrm{~nm}$ to $\Delta h_{\mathrm{eq}}\left(P_{n}^{\text {low }}\right)=0.4 \mathrm{~nm}$, i.e., a $2.1-\mathrm{nm}$ growth of the crystals. In accordance with the dependency $\Delta h_{\mathrm{eq}}\left(P_{n}\right)$, $\Delta h_{\mathrm{eq}}\left(P_{n}^{\text {low }}\right)$ is exactly the $a$-Si thickness the system would have reached had the simulation been performed at $P_{n}^{\text {low }}$ from the beginning.

While no net crystal growth occurs for symmetric tribopartners (i.e., the same crystal orientation and sliding direction), we now show that shear-induced growth of one crystal at the expense of the other can be achieved by breaking the symmetry of the sliding system. Figure 1(e) displays a $\mathrm{Si}(110)$ sliding system in which the lower crystal is rotated by $90^{\circ}$ with respect to the upper one, so that sliding proceeds along different crystallographic directions for the two crystals. Now, the lower crystal grows rapidly, as shown by the upward migration of $h_{1}$ and $h_{2}$ in Fig. 1(f), while $\Delta h$ remains roughly constant at $\Delta h_{\mathrm{eq}}$ (Movie S1 [20]). Growth is conveniently characterized by the rate $\xi(s)=d h_{1}(s) / d s$, which is $\sim 0.025$ and approximately constant in Fig. 1(f).

To elucidate the mechanism underlying the observed triboepitaxial growth, we first focus on the shear stress $\tau(s)$. 

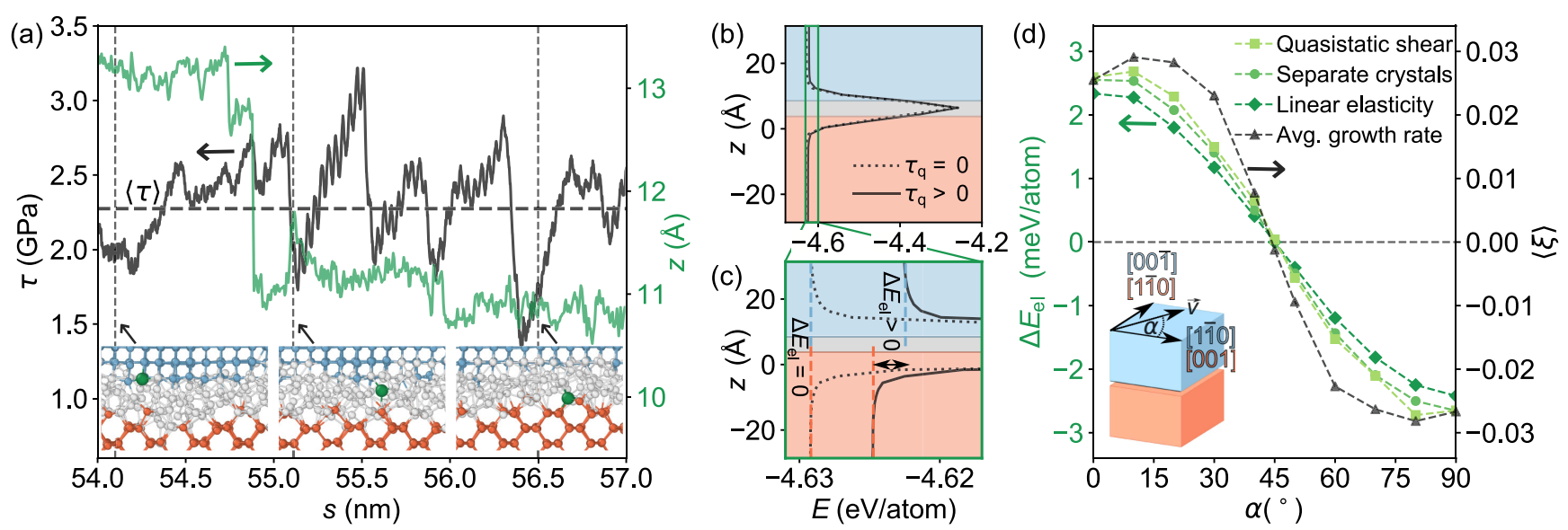

FIG. 2. Triboepitaxial growth mechanism. (a) Running average of shear stress $\tau$ (black) during the MD simulation in Fig. 1(f), showing stick-slip instabilities. The horizontal dashed line represents $\langle\tau\rangle$. The green line is the $z$ coordinate of an atom (green sphere) that is transferred from the upper to the lower crystal as a result of triboepitaxy. (b) Potential energy per atom $E$ as a function of $z$ for applied shear stress $\tau_{q}=0$ and $\tau_{q}>0$. (c) Enlargement with vertical dashed lines highlighting the energy levels of the crystals. (d) Elastic energy differences per atom $\Delta E_{\mathrm{el}}$ (green) between the upper and lower crystal when the original sliding direction [Fig. 1(e)] is rotated by an angle $\alpha$ (inset). $\Delta E_{\mathrm{el}}$ is determined by quasistatically shearing either the tribosystem (squares) or two single crystals (circles) and by linear elasticity (diamonds). Black triangles show the time-averaged growth rate of the lower crystal $\langle\xi\rangle$ measured in MD simulations (see definition in the main text).

Although the $a$-Si region migrates, the time-averaged shear stress $\langle\tau\rangle$ remains constant. Since also $\xi(s)$ and $\Delta h(s)$ remain roughly constant, we conclude that the properties of the crystal-amorphous-crystal transition region remain unaltered throughout the simulation. Figure 2(a) shows a sawtooth-shaped $\tau(s)$ characteristic of stick-slip instabilities. During stick phases, the system deforms elastically. When $\tau(s)$ exceeds the interfacial shear strength, the crystal-amorphous-crystal transition region undergoes plastic slip and its atoms gain mobility. In Fig. 2(a), we follow the typical trajectory of an atom directly involved in triboepitaxy during several stick-slip phases (Movie S2 [20]). Initially, the atom is part of the upper crystal. A series of plastic slip events cause the atom to be dragged into the $a$-Si region and to move to the lower amorphous-crystal interface, where it eventually recrystallizes.

To understand what drives the migration of the $a$-Si interface, we note that triboepitaxy resembles grain-boundary migration in metals under shear [32-34] and that thermally induced migration of grain boundaries under anisotropic elastic strain is driven by the elastic energy density difference $\Delta E_{\mathrm{el}}$ between grains $[35,36]$. We show in the following that $\Delta E_{\mathrm{el}}$ also determines the growth direction in shear-induced migration. An arbitrary steady-state configuration from the MD trajectory in Fig. 1(f) is selected and relaxed to $\tau=0 \mathrm{GPa}$ and $T=0 \mathrm{~K}$ (Supplemental Material [20]). This provides the starting structure for a quasistatic shear simulation where a finite shear stress $\tau_{q}>0$ is imposed by a stepwise displacement of the upper rigid layer in the lateral direction while continuously relaxing the atomic positions. The dotted and solid curves in Fig. 2(b) show the height dependence of the average potential energy per atom $E(z)$ at $\tau_{q}=0$ and $\tau_{q}=\langle\tau\rangle$ from Fig. 2(a). An enlargement [Fig. 2(c)] reveals that the energy density of both crystals is degenerate at $\tau_{q}=0$, while for $\tau_{q}>0$ the upper crystal atoms have a higher energy $\left(\Delta E_{\mathrm{el}} \approx 2.3 \mathrm{meV}\right)$. We propose that this gradient triggers the growth of the lower crystal.

We substantiate the existence of a clear correlation between $\Delta E_{\mathrm{el}}$ and the growth direction by additional sliding simulations where we rotate the sliding direction by an angle $\alpha \in\left(0^{\circ}, 90^{\circ}\right]$ [Fig. 2(d), inset]. Because of the symmetry of both crystals, the system is inversed at $\alpha=90^{\circ}$, where the shear directions of the lower and upper crystal are $[1 \overline{1} 0]$ and $[00 \overline{1}]$ (equivalent to [001]), respectively. The lower (upper) crystal grows for $\alpha<45^{\circ}$ $\left(\alpha>45^{\circ}\right)$ with approximately constant growth rate $\xi$. For $\alpha=45^{\circ}$, the two crystals slide along equivalent directions, and the time average $\langle\xi\rangle$ is zero. Importantly, $\Delta E_{\mathrm{el}}(\alpha)$ evaluated at the respective $\tau_{q}=\langle\tau\rangle$ closely correlates with $\langle\xi(\alpha)\rangle$ [Fig. 2(d)].

The condition $\Delta E_{\mathrm{el}} \neq 0$ results from an anisotropic elastic response of two misoriented crystals. The elastic energy density in a crystal under shear stress $\tau$ is given by $E_{\mathrm{el}}=\frac{1}{2} \tau^{2} / G^{*}$, where $G^{*}$ is the effective shear modulus. Because of crystal anisotropy, rotation changes $G^{*}$ in the sliding direction. Since $\tau$ is determined by plasticity of the $a$-Si phase and, hence, independent of this rotation, the change in shear modulus carries over directly to $\Delta E_{\mathrm{el}}$ between the two crystals. Consequently, $\Delta E_{\text {el }}$ can also be calculated by applying a simple shear deformation to the simulation cell of the individual crystals while relaxing the atomic positions and evaluating the potential energy differences per atom at $\langle\tau\rangle$ [Fig. 2(d) and Supplemental 
Material [20] ]. Alternatively, $\Delta E_{\mathrm{el}}$ from linear elasticity also shows good agreement [Fig. 2(d)] and requires only the elastic constants $C_{11}, C_{12}$, and $C_{44}$ of the cubic crystal.

We now turn to triboepitaxial growth with finite-sized tips on flat substrates. Figures 3(a) and 3(b) show snapshots of $\mathrm{Si}(110)$ tips after sliding for $s \approx 600 \mathrm{~nm}$ along their [001] direction on infinite $\mathrm{Si}(110)$ (along [11̄0]; see Supplemental Material [20]). According to Fig. 2(d), the tips should grow, and, indeed, the tip with width $l \approx 151 \AA$ follows this prediction [Fig. 3(a) and Movie S3 [20] ]. Surprisingly, for $l \approx 53 \AA$ the substrate grows by $\sim 4$ atomic layers [Fig. 3(b) and Movie S4 [20] ]. This can be ascribed to the strong size dependence of the effective elastic response of nanosized structures [37-40]. To determine the triboepitaxial growth direction, we have to consider the ordering of $G_{\text {substrate }}^{*}$ and $G_{\text {tip }}^{*}$, as these two effective shear moduli determine the average elastic energy density in each of the two crystals and, hence, $\Delta E_{\mathrm{el}}$. We perform quasistatic shear deformations of tips of various length $l$ to obtain their shear moduli $G^{*}(l)$ [Fig. 3(c) and Supplemental Material [20] ]. For large $l, G^{*}$ slowly converges to the value of an infinite (110) surface $G^{*}(\infty)$, while it rapidly decreases for $l<20 \mathrm{~nm}$. In analogy to Ref. [37], we formulate an analytical expression for $G^{*}(l)$ by separating $G^{*}$ into a surface contribution $G^{*}(2 b)$, defined by a surface thickness $b$ [Fig. 3(c), inset], and a bulk contribution $G^{*}(\infty)$. The analytical $G^{*}(l)$ given by the weighted average

$$
G^{*}(l)=G^{*}(\infty) \frac{l-2 b}{l}+G^{*}(2 b) \frac{2 b}{l}
$$

follows closely the quasistatic shearing results. Equation (1) suggests that an increase in the surface-tovolume ratio underlies the anomaly in triboepitaxial growth observed for the small tip in Fig. 3(b). Indeed, since $G^{*}(l=151 \AA)>G_{\text {substrate, }}^{*}$ the large tip grows, while $G^{*}(l=53 \AA)<G_{\text {substrate }}^{*}$ determines epitaxial growth on the substrate. Thus, the tip size represents an additional control variable for triboepitaxy.

Finally, we propose an experimental setup for triboepitaxy [Fig. 3(d)]. A (110)-oriented nanoscale tip (blue) oscillates with velocity $\vec{v}_{\text {osc }}$ along its $[1 \overline{1} 0]$ direction and rubs on a Si(110) substrate (red) along the [001] direction. The choice of these orientations favors the growth of the substrate irrespective of the tip size. Further simulations reveal that a variety of surface orientations can be used and that the process is robust with respect to changes in $T$ and $P_{n}$ (Supplemental Material [20]). Typically, in experiments $v_{\text {osc }}$ is about $100 \mathrm{~nm} / \mathrm{s}[41,42]$. If $\langle\xi\rangle \sim 0.01$ [Fig. 2(d)], we expect a growth velocity $\xi v_{\text {osc }}$ of about $1 \mathrm{~nm} / \mathrm{s}$. The introduction of an additional translation $\vec{v}_{\text {trans }}(t)$, with $v_{\text {trans }} \ll v_{\text {osc }}$, enables writing of arbitrary crystalline nanolines. We note that the magnitude of the tip oscillation must be large enough to ensure that the critical shear stress for plastic deformation [Fig. 2(a)] is reached at the tip-substrate interface. Its value depends on the tip's geometry and
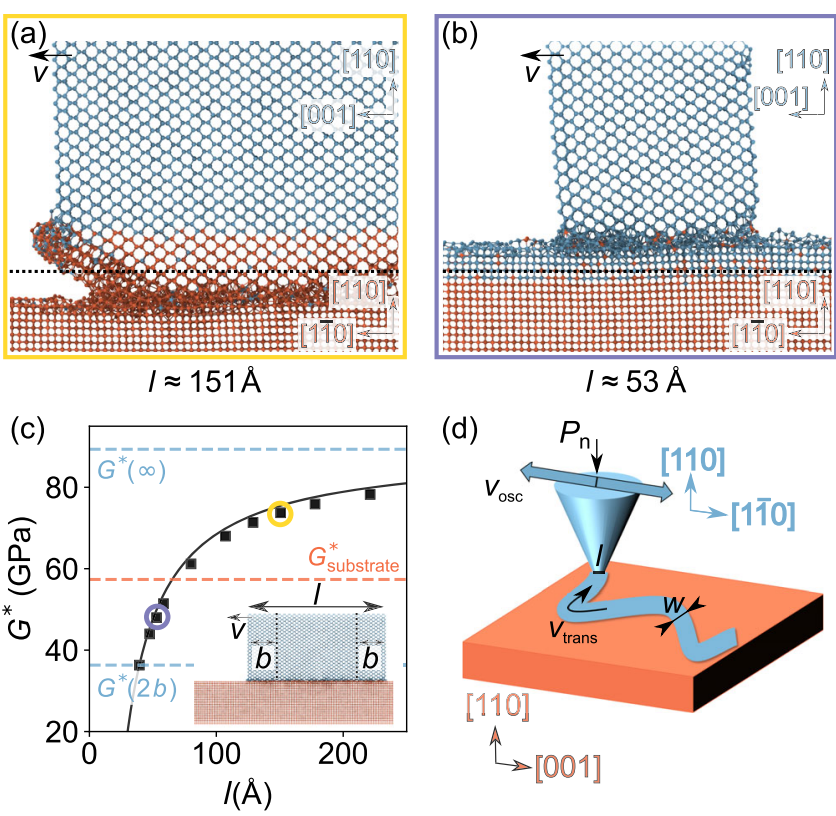

FIG. 3. Triboepitaxy with a finite tip. (a),(b) Snapshots of sliding simulations with tip sizes $l \approx 151 \AA$ and $l \approx 53 \AA$ at $s \approx$ $600 \mathrm{~nm}$ along [001] of the tip and [110] of the substrate. The horizontal dashed lines indicate the initial position of the interface between tip (blue) and substrate (red). (c) Effective shear modulus $G^{*}(l)$ of the tips. The tip's width $l$ is shown in the inset along with the surface thickness $b$, which is the thickness of the tip's surface region in which the elastic response differs from the bulk elastic response, i.e., the response of the infinite tip $(l=\infty)$. Black squares denote $G^{*}$ from quasistatic shear deformation. Dashed horizontal lines indicate $G^{*}$ of an infinite substrate $\left(G_{\text {substrate }}^{*}\right)$ and tip $\left[G^{*}(\infty)\right]$ and of a tip whose elastic response is completely determined by its surface $\left[G^{*}(2 b)\right]$. The solid curve shows the interpolation Eq. (1) between bulk and surface modulus. The best fit to the data points is obtained using $b \approx 19.68 \AA$ (Supplemental Material [20]). (d) Scheme for an experiment to deposit silicon nanostructures on (110) silicon substrates using (110) tips. Highfrequency oscillations with velocity $\vec{v}_{\text {osc }}$ along [1 $\left.1 \overline{1} 0\right]$ of the tip and [001] of the substrate induce triboepitaxial growth. For writing, the tip is slowly translated with a velocity $\vec{v}_{\text {trans }}(t)$ along arbitrary directions, leaving behind a nanocrystalline line with width $w=l+w_{\text {osc }}$, where $w_{\text {osc }}$ is the magnitude of tip oscillation at the contact. Besides the oscillation direction $\hat{v}_{\text {osc }}$, also surface orientation, $l$, and $P_{n}$ influence triboepitaxial growth (Supplemental Material [20]).

stiffness. Typical reciprocating strokes used to study wear of atomic force microscopy tips are about $100 \mathrm{~nm}$ [42], which is necessarily larger than the width of the deposited crystalline film, because it also includes the residual elastic deformation of the tip [minimum $\tau$ is larger than zero in Fig. 2(a)].

In conclusion, the triboepitaxy concept presented in this work provides an example of how the liquidlike behavior [10] caused by large shear plastic deformation in a nanoscale $a$-Si shear band can enable a thermodynamically driven crystal growth process at temperatures that are 
significantly below those usually required for solid-state crystallization of $a-\mathrm{Si}$ [15]. The idea could be tested on other materials showing shear-induced amorphization (e.g., diamond [11]) or materials on which solid-state epitaxy was applied successfully (e.g., germanium [15]). Since triboepitaxial growth is simply governed by the elastic properties of the two sliding crystals, predictions on the crystal growth direction are straightforward, and setting up an experiment to verify the results of our simulations should be possible. This could be attempted by using atomic force microscopy probes inside a transmission electron microscope [41]. Adhesion experiments performed under these conditions suggest that surfacepassivating species that prevent the formation of covalent bonds across the tribological interface are easily removed by sliding [41]. Moreover, the presence of surface defects should not be critical, since the process relies on surface amorphization in the first place. To further investigate the generality of the proposed mechanisms, simulations are underway to investigate triboepitaxy at sliding contacts between two differently oriented surfaces (Supplemental Material [20]). In such cases, due to the different elastic responses of the two crystals to normal pressure, the applied normal force can become an additional parameter to control crystal growth direction and rate. Finally, this study suggests a new way to apply tribological concepts in the context of nanolithography and nanofabrication. While mechanical scanning-probe techniques are usually based on nanomachining [17,43-45], i.e., removal of material from a surface or surface amorphization, we propose that tribologically induced phase transitions could be exploited for direct deposition of nanostructures or selective microstructural modification of a surface.

We are grateful to William Curtin for helpful discussions and to Andreas Klemenz and Hiroshi Uetsuka for initial contributions to the project. We gratefully acknowledge the Gauss Centre for Supercomputing e.V. for funding this project by providing computing time through the John von Neumann Institute for Computing (NIC) on the GCS Supercomputer JUWELS [46], at Jülich Supercomputing Centre (JSC). We also acknowledge computing time by the state of Baden-Württemberg through bwHPC and the German Research Foundation (DFG) (Grant No. INST 39/963-1 FUGG, bwForCluster NEMO). L. P. acknowledges support from the DFG (Grant No. PA 2023/2).

*Corresponding author. gianpietro.moras@iwm.fraunhofer.de

[1] A. Kailer, Y. G. Gogotsi, and K. G. Nickel, J. Appl. Phys. 81, 3057 (1997).

[2] J. R. Kermode, T. Albaret, D. Sherman, N. Bernstein, P. Gumbsch, M. C. Payne, G. Csányi, and A. De Vita, Nature (London) 455, 1224 (2008).
[3] F. Östlund, K. Rzepiejewska-Malyska, K. Leifer, L. M. Hale, Y. Tang, R. Ballarini, W. W. Gerberich, and J. Michler, Adv. Funct. Mater. 19, 2439 (2009).

[4] Y. He, L. Zhong, F. Fan, C. Wang, T. Zhu, and S. X. Mao, Nat. Nanotechnol. 11, 866 (2016).

[5] A. Merabet, M. Texier, C. Tromas, S. Brochard, L. Pizzagalli, L. Thilly, J. Rabier, A. Talneau, Y. M. Le Vaillant, O. Thomas, and J. Godet, Acta Mater. 161, 54 (2018).

[6] J. R. Kermode, L. Ben-Bashat, F. Atrash, J. J. Cilliers, D. Sherman, and A. De Vita, Nat. Commun. 4, 2441 (2013).

[7] G. Moras, L. Colombi Ciacchi, C. Elsässer, P. Gumbsch, and A. De Vita, Phys. Rev. Lett. 105, 075502 (2010).

[8] M. Chen, L. Pethö, A. S. Sologubenko, H. Ma, J. Michler, R. Spolenak, and J. M. Wheeler, Nat. Commun. 11, 2681 (2020).

[9] X. D. Han, K. Zheng, Y. F. Zhang, X. N. Zhang, Z. Zhang, and Z. L. Wang, Adv. Mater. 19, 2112 (2007).

[10] G. Moras, A. Klemenz, T. Reichenbach, A. Gola, H. Uetsuka, M. Moseler, and L. Pastewka, Phys. Rev. Mater. 2, 083601 (2018).

[11] L. Pastewka, S. Moser, P. Gumbsch, and M. Moseler, Nat. Mater. 10, 34 (2011).

[12] S. Goel, A. Kovalchenko, A. Stukowski, and G. Cross, Acta Mater. 105, 464 (2016).

[13] B. Fuhrmann, H. S. Leipner, H.-R. Höche, L. Schubert, P. Werner, and U. Gösele, Nano Lett. 5, 2524 (2005).

[14] H. Kum, D. Lee, W. Kong, H. Kim, Y. Park, Y. Kim, Y. Baek, S. Bae, K. Lee, and J. Kim, Nat. Electron. 2, 439 (2019).

[15] B. C. Johnson, J. C. McCallum, and M. J. Aziz, in Handbook of Crystal Growth, 2nd ed., edited by T. F. Kuech (Elsevier, North-Holland, 2015), pp. 317-363, https://doi .org/10.1016/B978-0-444-63304-0.00007-X.

[16] J. W. Park, N. Kawasegi, N. Morita, and D. W. Lee, Appl. Phys. Lett. 85, 1766 (2004).

[17] R. Garcia, A. W. Knoll, and E. Riedo, Nat. Nanotechnol. 9, 577 (2014).

[18] L. Pastewka, A. Klemenz, P. Gumbsch, and M. Moseler, Phys. Rev. B 87, 205410 (2013).

[19] T. Kumagai, S. Izumi, S. Hara, and S. Sakai, Comput. Mater. Sci. 39, 457 (2007).

[20] See Supplemental Material at http://link.aps.org/ supplemental/10.1103/PhysRevLett.127.126101 for computational methods; interatomic potentials; overview of the systems used for the simulations of periodic interfaces; role of strain due to lattice mismatch in periodic simulations; dependence of triboepitaxy on the sliding velocity; $\Delta E_{\mathrm{el}}$ as a function of the sliding distance; generalization to other surface orientations; robustness of triboepitaxy with respect to temperature and pressure variations; analytical model for the effective shear modulus $G^{*}(l)$ of the tip; and descriptions of movies, which includes Refs. [21-28].

[21] L. Pastewka, S. Moser, and M. Moseler, Tribol. Lett. 39, 49 (2010).

[22] A. Stukowski, Model. Simul. Mater. Sci. Eng. 18, 015012 (2010).

[23] E. Maras, O. Trushin, A. Stukowski, T. Ala-Nissila, and H. Jónsson, Comput. Phys. Commun. 205, 13 (2016).

[24] S. Plimpton, J. Comput. Phys. 117, 1 (1995). 
[25] A. H. Larsen et al., J. Phys. Condens. Matter 29, 273002 (2017).

[26] R. Drosd and J. Washburn, J. Appl. Phys. 53, 397 (1982).

[27] E. Lampin and C. Krzeminski, J. Appl. Phys. 106, 063519 (2009).

[28] G. Singh, J. R. Kermode, A. De Vita, and R. W. Zimmerman, Int. J. Fract. 189, 103 (2014).

[29] F. H. Stillinger and T. A. Weber, Phys. Rev. B 31, 5262 (1985).

[30] A. P. Bartók, J. Kermode, N. Bernstein, and G. Csányi, Phys. Rev. X 8, 041048 (2018).

[31] V. L. Deringer, N. Bernstein, G. Csányi, C. Ben Mahmoud, M. Ceriotti, M. Wilson, D. A. Drabold, and S. R. Elliott, Nature (London) 589, 59 (2021).

[32] J. W. Cahn, Y. Mishin, and A. Suzuki, Acta Mater. 54, 4953 (2006).

[33] T. J. Rupert, D. S. Gianola, Y. Gan, and K. J. Hemker, Science 326, 1686 (2009).

[34] V. A. Ivanov and Y. Mishin, Phys. Rev. B 78, 064106 (2008).

[35] H. Zhang, M. I. Mendelev, and D. J. Srolovitz, Acta Mater. 52, 2569 (2004).
[36] B. Schönfelder, D. Wolf, S. R. Phillpot, and M. Furtkamp, Interface Sci. 5, 245 (1997).

[37] J. Q. Broughton, C. A. Meli, P. Vashishta, and R. K. Kalia, Phys. Rev. B 56, 611 (1997).

[38] R. E. Miller and V. B. Shenoy, Nanotechnology 11, 139 (2000).

[39] X. Li, T. Ono, Y. Wang, and M. Esashi, Appl. Phys. Lett. 83, 3081 (2003).

[40] S. H. Park, J. S. Kim, J. H. Park, J. S. Lee, Y. K. Choi, and O. M. Kwon, Thin Solid Films 492, 285 (2005).

[41] Z. B. Milne, R. A. Bernal, and R. W. Carpick, Langmuir 35, 15628 (2019).

[42] T. D. B. Jacobs and R. W. Carpick, Nat. Nanotechnol. 8, 108 (2013).

[43] B. Yu, H. Dong, L. Qian, Y. Chen, J. Yu, and Z. Zhou, Nanotechnology 20, 465303 (2009).

[44] A. A. Tseng, Small 7, 3409 (2011).

[45] L. Chen, J. Wen, P. Zhang, B. Yu, C. Chen, T. Ma, X. Lu, S. H. Kim, and L. Qian, Nat. Commun. 9, 1542 (2018).

[46] Jülich Supercomputing Centre, J. Large-Scale Res. Facil. 5, A135 (2019). 